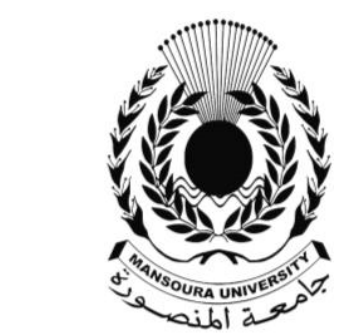

MANSOURA UNIVERSITY

FACULTY OF ARTS

\title{
ZWEI DENKMÄLER AUS UNBEKANNTER HERKUNFT VON
}

\author{
By
}

\section{Dr. Nehad Kamal Eldin}

Diese Untersuchung behandelt zwei

Denkmäler, die weit von ihrem originalen

Platz gefunden wurden und bis jetzt ist ihr originaler Platz unbestimmt. 


\title{
ZWEI DENKMÄLER AUS UNBEKANNTER HERKUNFT VON
}

\author{
Dr. Nehad Kamal Eldin \\ Diese Untersuchung behandelt zwei \\ Denkmäler, die weit von ihrem originalen
}

Platz gefunden wurden und bis jetzt ist ihr originaler Platz unbestimmt.

\section{Das Erste Denkmal}

Das Erste Denkmal ist ein Architrav, der den Namen des Königs Nb mAat Ra Amenophis III trägt. Seine Herkunft ist unbestimmt, und er befindet sich heute im Abstellraum der Pyramiden ohne Registrationsnummer ${ }^{1}$. Dieser Architrav ist unveröffentlicht. Ein altes Bild von diesem Architraven erscheinte in der schriftlichen Arbeit von Moursi ${ }^{2}$ (Abb.1). Er sagte, dass dieser Architrav in Tell-Hisn war, und er sieht, dass er ein Teil vom alten Tempel des Amenophis III, der südlich des Temples von Ramses IX stand, ist.

Es ist unklar, wann und wie dieser Architrav nach Gisa gebracht wurde, wie er zerbrochen wurde und ob Tell-Hisn sein originaler Platz ist. Oder wurde er danach von einem anderen Platz gebracht.

Im Folgenden beschreibe ich diesen Architrav und übersetze seine Texte

Ich werde auch zwischen dem alten Bild dieses Architravs und seinem aktuellen Zustand vergleichen.(Abb.2,3)

\subsection{Beschreibung}

Der Architrav ist aus Kalkstein angefertigt und ist $\mathrm{ca} 170 \mathrm{~cm}$ hoch, $80 \mathrm{~cm}$ breit und 40 $\mathrm{cm}$ dick. Obwohl er in einem schlechten Zustand ist, weist er Bemalungsspuren auf. Dieser Architrav ist zerbrochen. Er ist vielleicht ein Teil einer grossen Wand. Er ist von einer Hohlkehle gekrönt. Darunter befindet sich ein Rundstab, der die Wand einrahmt. Die Darstellungen und Inschriften sind in versinktem Relief gearbeitet.

\subsection{Darstellungen und Inschriften}

Auf der Hohlkehle befinden sich viele Kartuschen, die den Namen Nb mAat Ra enthalten.

Darunter gibt es die zentrale Szene, die drei gabenbringende Gottheiten und Nb mAat $\mathrm{Ra}$ als Name des kgl. Empfängers zeigt. Diese Szene ist Teil einer größeren fehlenden Szene, die eine Reihe von gabenbringenden Gottheiten darstellt ${ }^{3}$. Die drei Götter wenden sich nach links. Die erste von ihnen ist die Göttin Nbt-KAw, die Herrin der Speisen ${ }^{4}$. Sie erscheint als Frau mit einem Opfertablett in ihren Händen und Kälbchen vor ihren Füßen.

Vor ihr befinden sich zwei vertikale Zeilen ${ }^{5}$ von Inschriften, von denen nur eine bleibt. Der Text liest sich folgendermaßen von links nach rechts:

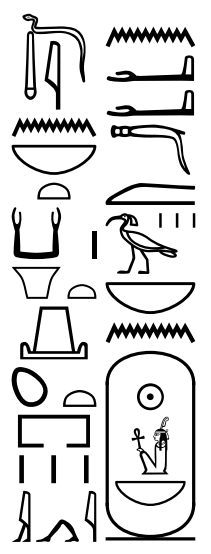

[Dd mdw in nbt kAw Hnwt Snwt ${ }^{6}$ ii].n.(i) awj.(i) $\mathrm{mH} m$ DfAw nb $\mathrm{n}$ nb mAat $\mathrm{Ra}$

[Worte sprechen seitens von Nebet Kaw, die Herrin der Kornkammer. Ich bin gekommen]. Meine Arme gefüllt mit all Speisen $^{7}$ für Nb mAat $\mathrm{Ra}$ 
Der zweite Gott ist der Nilgott $\mathrm{Hapi}^{8}$. Er erscheint als Mann mit weiblicher Brust, und fettem Leib, der Wülste bildet und über den Leibgurthinabhängt. Er trägt einen Gürtel mit befestigten Zeugstreifen und einen Götterbart. In seinen Händen hält er Büschel von Papyruspflanzen ${ }^{9}$ Die begleitende Inschrift vor ihm verteilt sich folgendermaßen in zwei vertikale Zeilen:

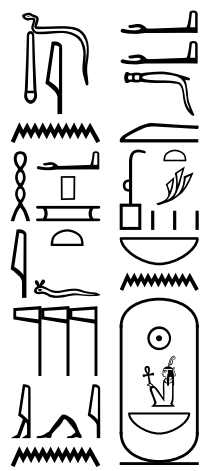

Dd mdw in Hapi it nTrw ii.n.(i) awj (i) $\mathrm{mH} m$ rnpwt nb $n$ Nb mAaT Ra

"Worte sprechen seitens von Hapi, der Vater der Götter ${ }^{10}$. Ich bin gekommen meine Arme gefüllt mit verschiedenen frischen Pflanzen ${ }^{11}$ für No MAat Ra"

Die dritte und letzte Göttin ist die Göttin Nenut $^{12}$. Sie trägt Brot und Papyruspflanzen auf der Opferplatte. In ihrer rechten Hand hängt sie auch eine Papyruspflanze. Auf ihrem Kopf trägt sie einen Napf, den sie für die Reinigung benutzt. Vor ihr befindet sich ein voller Brotkorb. Die begleitende Inschrift vor ihr verteilt sich in zwei vertikale Zeilen und liest sich folgendermaßen von links nach rechts:

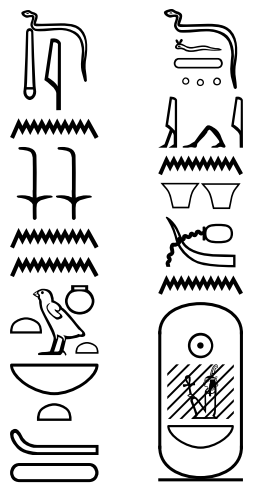

Dd mdw in Nnwt nbt $\mathrm{Hw} \mathrm{DfAw}^{1}$ ii.n.i awj.i wab $n$ Nb MAat Ra

"Worte sprechen seitens von Nenut, die Herrin von Speise und Nahrung. Ich bin gekommen, meine $\mathrm{Arme}^{14}$ sind rein für $\mathrm{Nb}$ MAat Ra".

Diese Szene ist auf der rechten Seite fortgesetzt, wo die Reste der Gaben und Blumen betrachtet werden können (Abb.4,5)

Auf der Außenseite dieser rechten Seite wird der Gott Hapi nochmal dargestellt.

Er erscheint als Mann mit weiblicher Brust, dessen fetter Leib Wülste bildet, die schwer über den Leibgurthinabhängen. Er trägt einen Götterbart, einen Gürtel befestigte Zeugstreifen, und eine Perücke. Auf seinem Kopf trägt er ein Büschel Papyruspflanzen und in beiden Händen hält er Gänse $^{15} \cdot($ Abb.6,7)

\subsection{Kommentar}

Wir sind nicht sicher, ob dieser Architrav aus Tell-Hisn stammt, wie Moursi ${ }^{16}$ sagte, oder nicht.

Die Szene der gabenbringenden Götter war weitverbreitet in der achtzehnten Dynastie, insbesondere der Zeit des Königs Amenophis III.

Es gibt eine andere Szene der gabenbringenden Götter von seiner Zeit auf einem Steinblock,der aus Kom el Ahmer stammt,und Jetzt im Cleveland Museum ausgestellt wird ${ }^{17}$.

Vielleicht stammte dieser Architrav von dem gleichen Platz und wurde nach TellHisn, wo Moursi ihn sah, gebracht. Danach wurde er nach Gisa gebracht, wo er heute liegt.

\section{Das zweite Denkmal}

Das zweite ist ein Steinblock aus der Zeit des Königs Apris(Abb.8,9). Es stammt aus einem kleinen Dorf, was Met el-laith Hashim heißt und in der Nähe von Mahalla al-Kubra ist. Er befindet sich heute in der Ablage. Er ist unveröffentlicht ${ }^{18}$. 


\subsection{Beschreibung:}

Der rechteckige Block ist aus Sandstein angefertigt und ist $\mathrm{ca} .41 \mathrm{~cm}$ hoch, $38 \mathrm{~cm}$ breit und $33 \mathrm{~cm}$ dick. Im oberen Teil des Blockes befindet sich ein Schlitz, sein Durchmesser ist $28 \mathrm{~cm}$ und seine Tiefe ist 38 cm. Dieser Schlitz ergab sich aus der Weiterverwendung des Blockes als Schleifstein (Abb.10,11). Der Block ist in einem gut erhaltenen Zustand. Die Darstellungen und Inschriften sind in versinktem Relief gearbeitet. Sein Typ ist charakterstich für die Blöcke, die zu Sais gehören.

\subsection{Inschriften}

Auf einer Seite dieses Stückes befindet sich eine kurze Inschrift. Sie wird in zwei Teile, durch das Pt Zeichen, geteilt, der obere Teil weist Spuren von hieroglyphischen Zeichen auf, das linke Zeichen weist einen Rest des Wortes Dt auf, während das rechte unklar ist. Es ist vielleicht der untere Teil des Zeichen ? $^{2} 108^{19}$. Im unteren Teil befinden sich drei senkrechte Kolumnen, die den Titel und Namen des König WAH ib Ra in einer Kartusche enthalten.

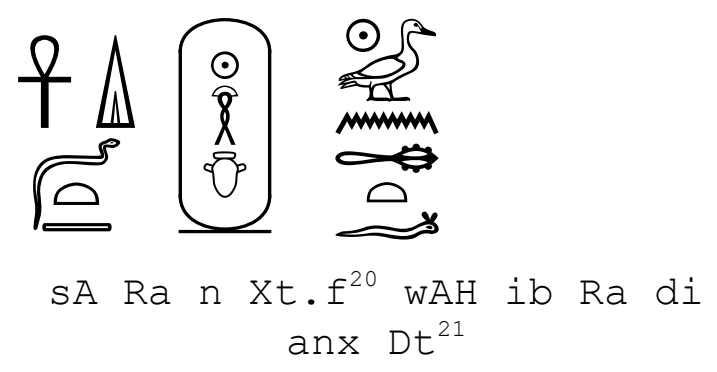

"Der Sohn des Ra, von seinem Körper WAH - Ib- Ra, möge er ewig leben".

\subsection{Darstllungen}

Unter dem Text befindet sich ein Kopf der König Apris. Er wendet sich nach rechts und trägt das Nemes-Kopftuch, von dem nur der hintere zusammengebundende Teil und der rechte Streifen bleibt. Das Gesicht des Königs und die Uräusschlange auf seinem Stern sind beschädigt. Die Darstellung des Königs und die Inschriften darüber werden von einem Rundstab eingerahmt. Über dem Kopf und der dreizeiligen Inschrift darüber befindet sich ein Himmelzeichen trennt diese Inschrift von der gebrochenen Inschrift darüber.

\subsection{Kommentar}

Obwohl dieser Block weit weg von Sais gefunden wurde, zeigt sein Typ, dass er zu Sais gehört. ${ }^{22}$

Er ist von der Zeit des Königs Apris von der Sechsundzwanzigsten Dynastie datiert, der in Sais begraben wurde ${ }^{23}$.

Er ist vielleicht ein oberer Teil der Wand oder des Pfostens. Das Himmelzeichen über dem König erklärt, dass diese Szene der obere Teil einer Szene ist, die den König vor verschiedenen Göttern darstellt. Er gibt ihnen Opfer und sie verleihen ihm ihre Segen. 


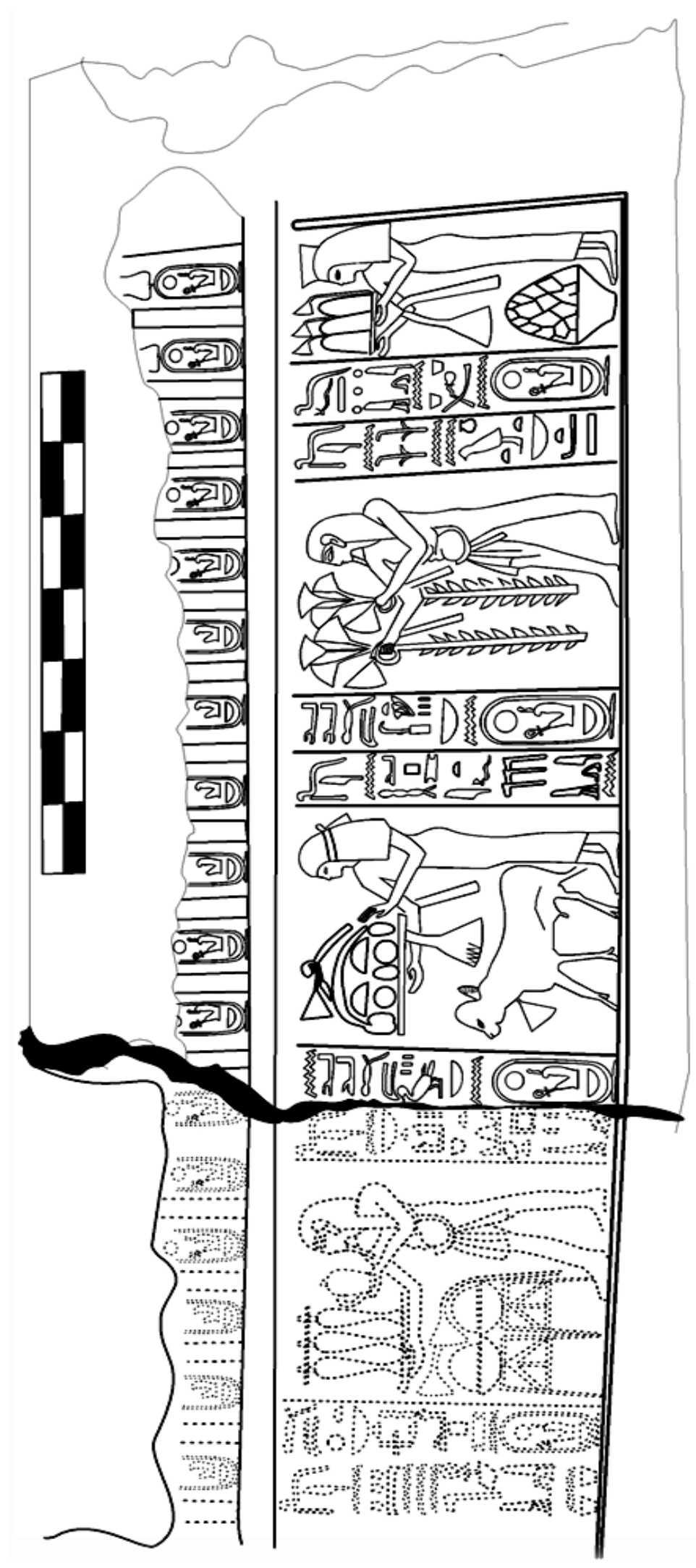

Abb.1

Ein Malen des Architravs vor dem Bruch im 1972.

M.Moursi, Die Hohepriester des Sonnengottes,MÄS 26(München,1972).78 Taf. XIII 


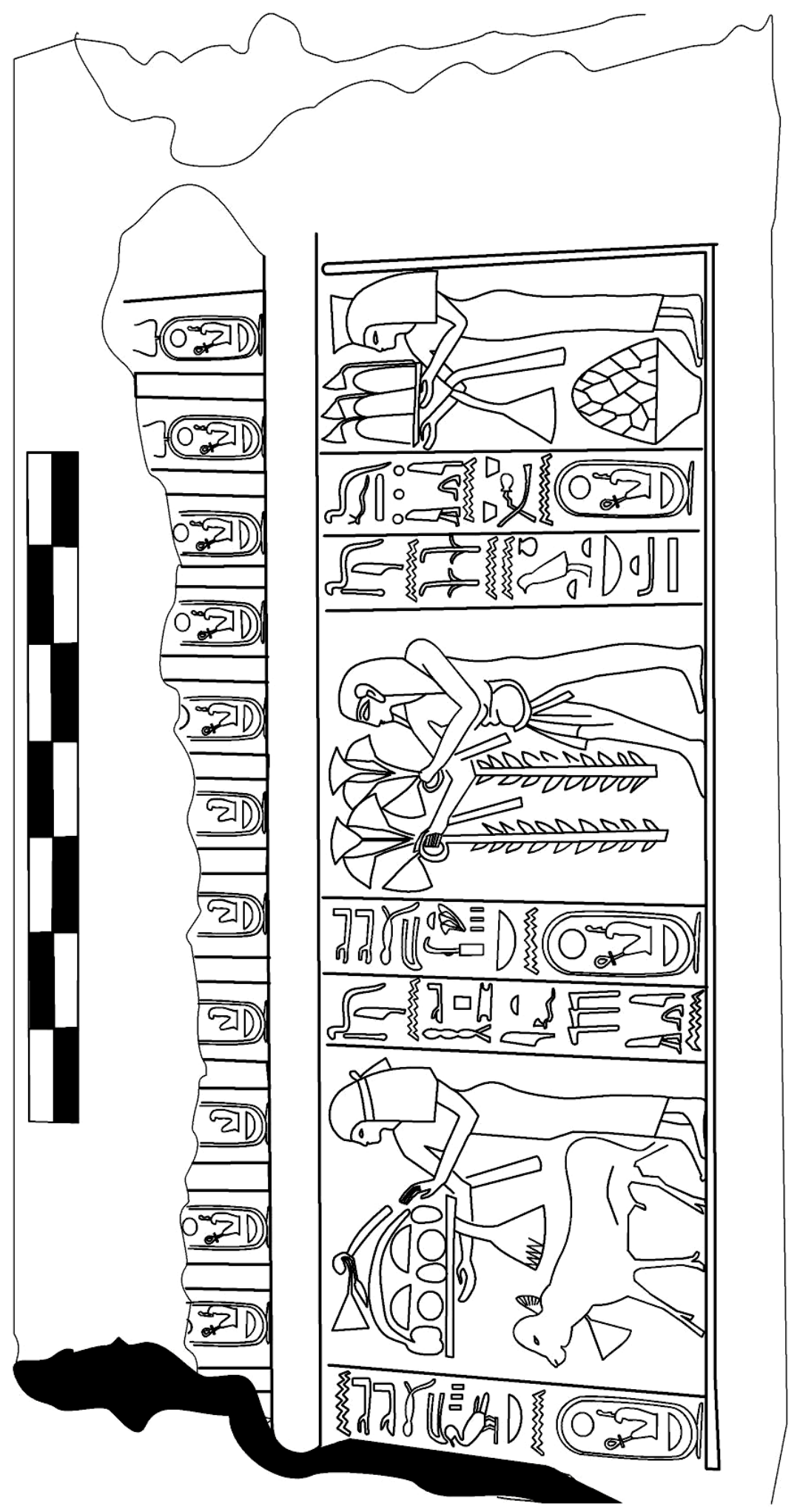

Abb.2 Das aktuelle Bild des Architravs 


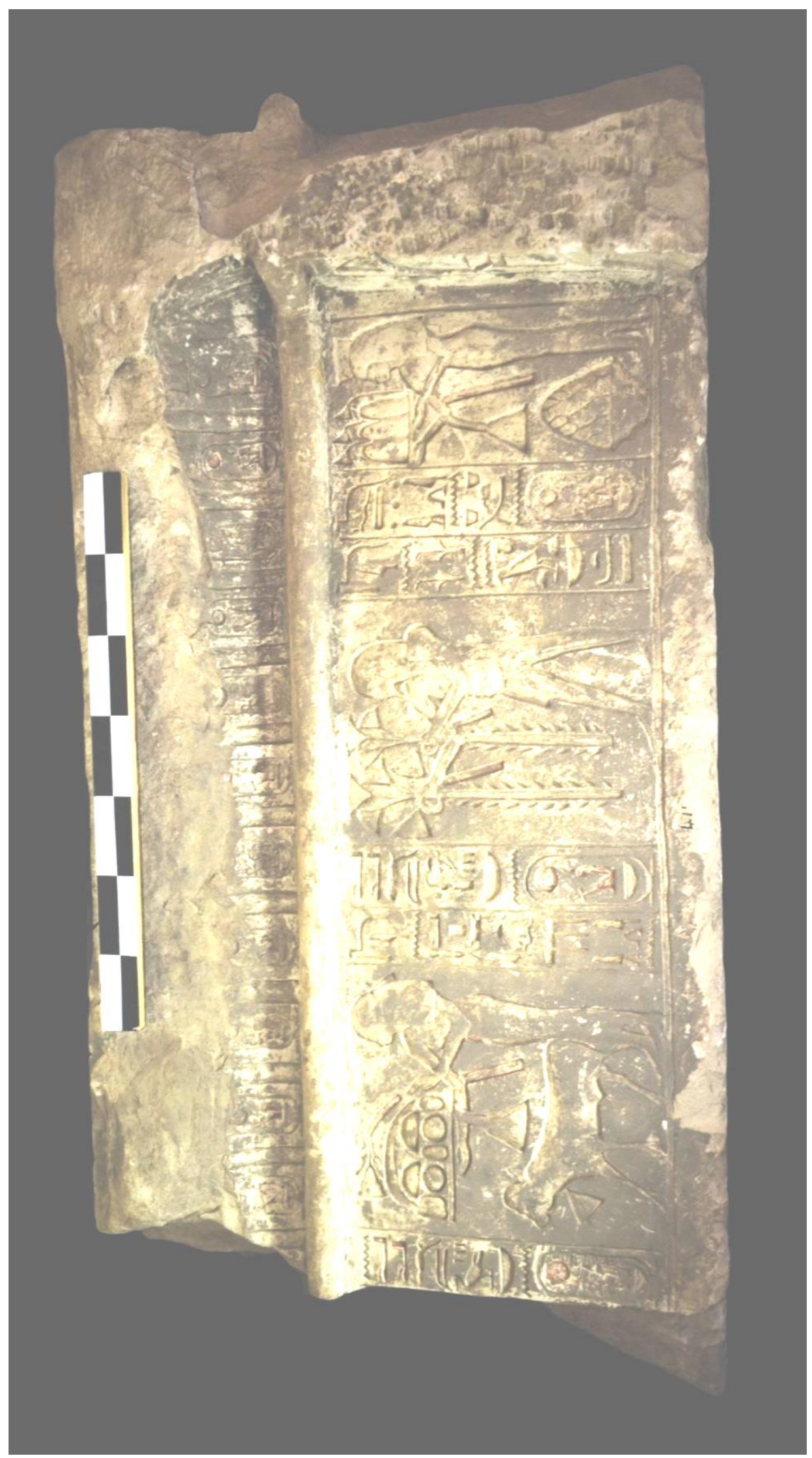

Abb.3 Das aktuelle Bild des Architravs 


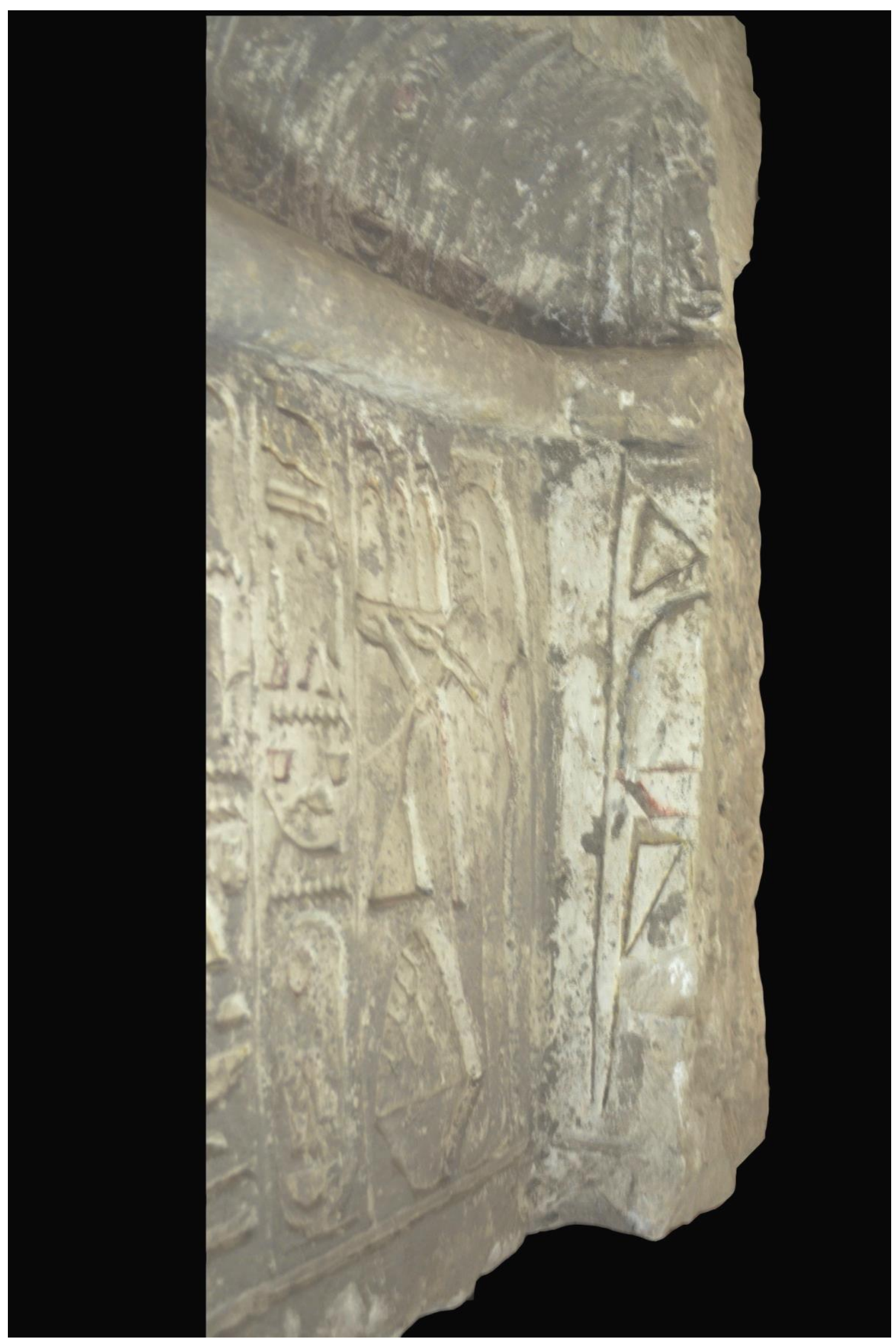

Abb.4 Die Darstellung auf der rechten Seite von innen 


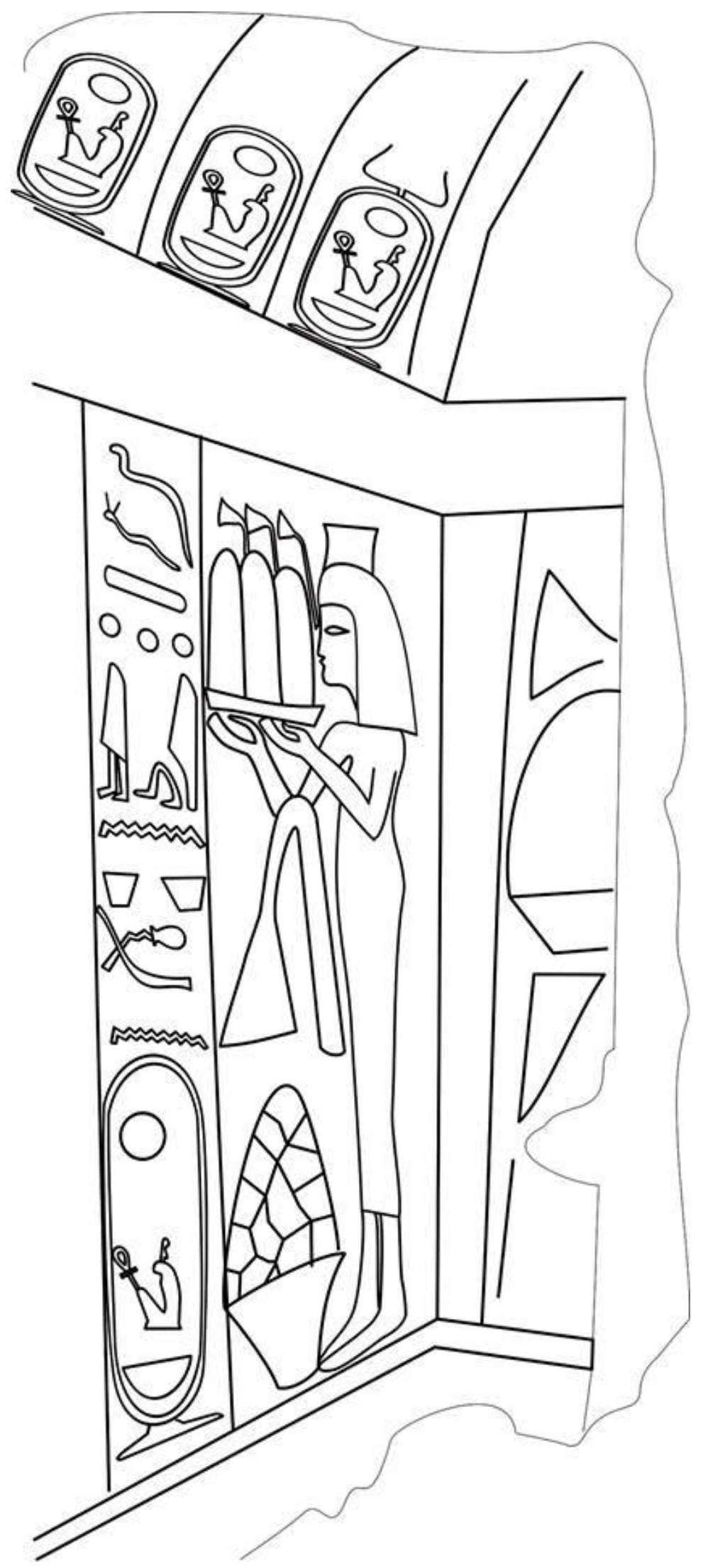

Abb.5 Ein Malen zeigt die Darstellung auf der rechten Seite von innen 


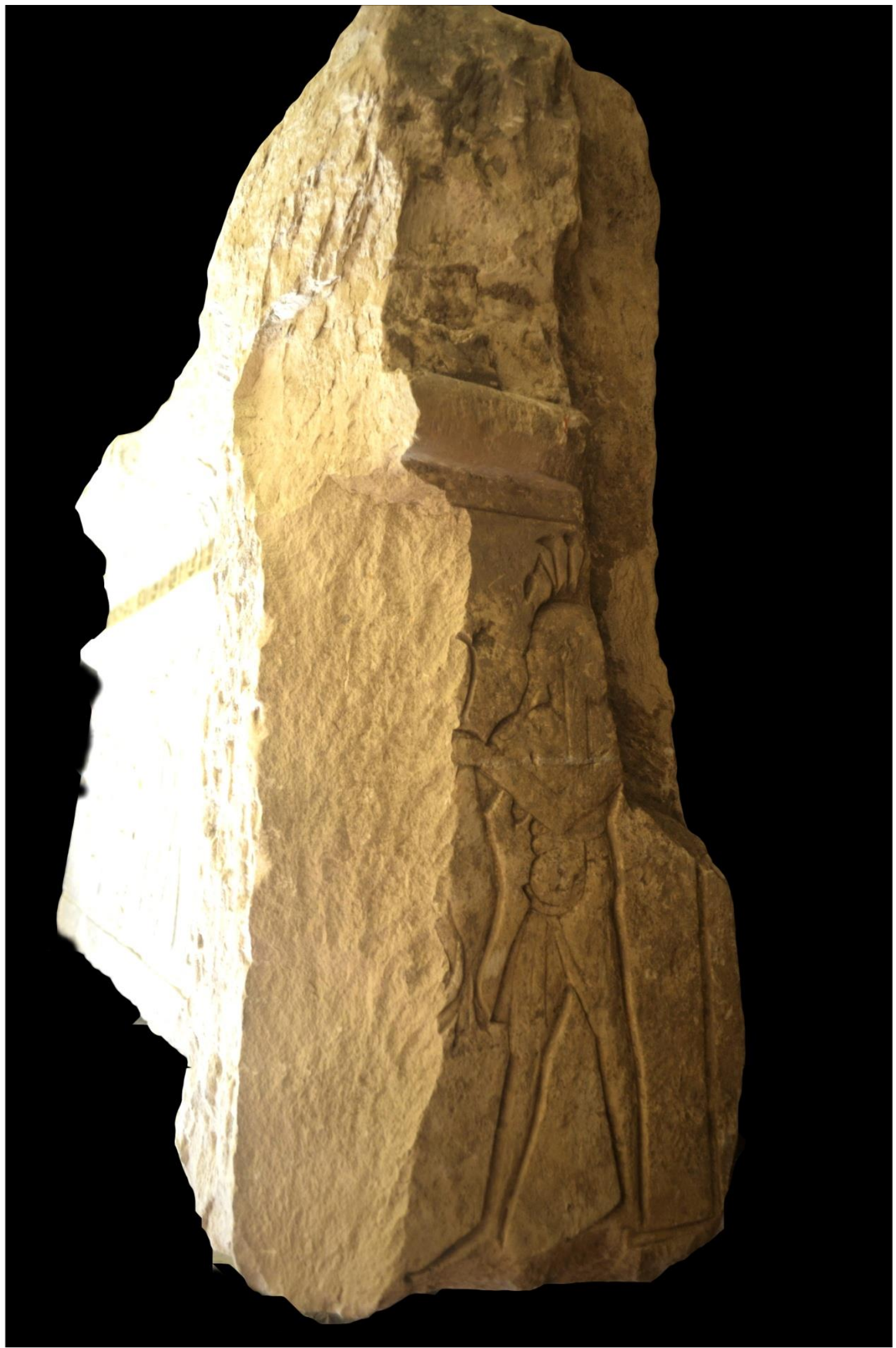

Abb.6 Die Darstellungen auf der rechten Seite von außen 


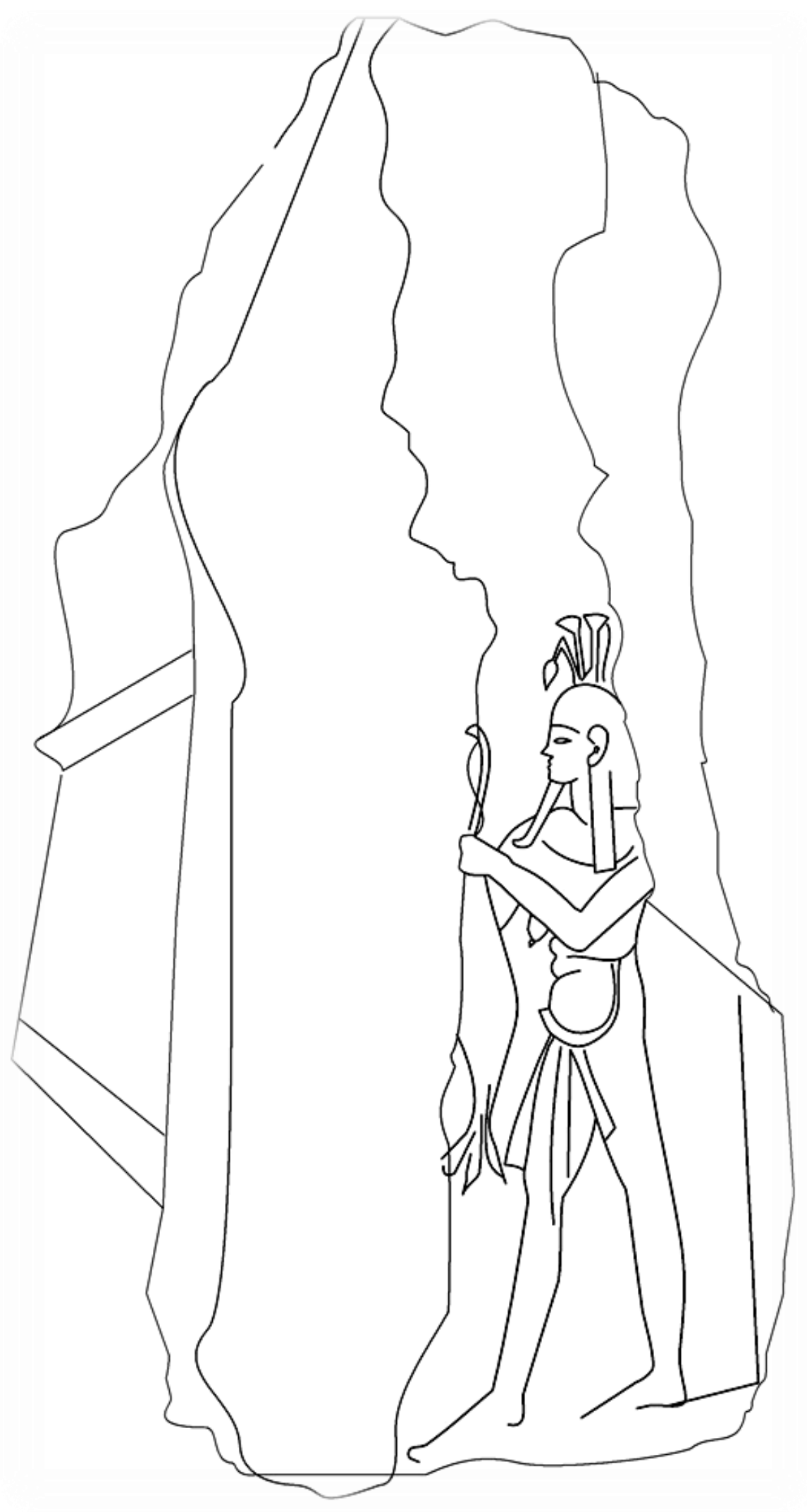

Abb.7 Ein Malen der Darstellung auf der rechten Seite Von außen 


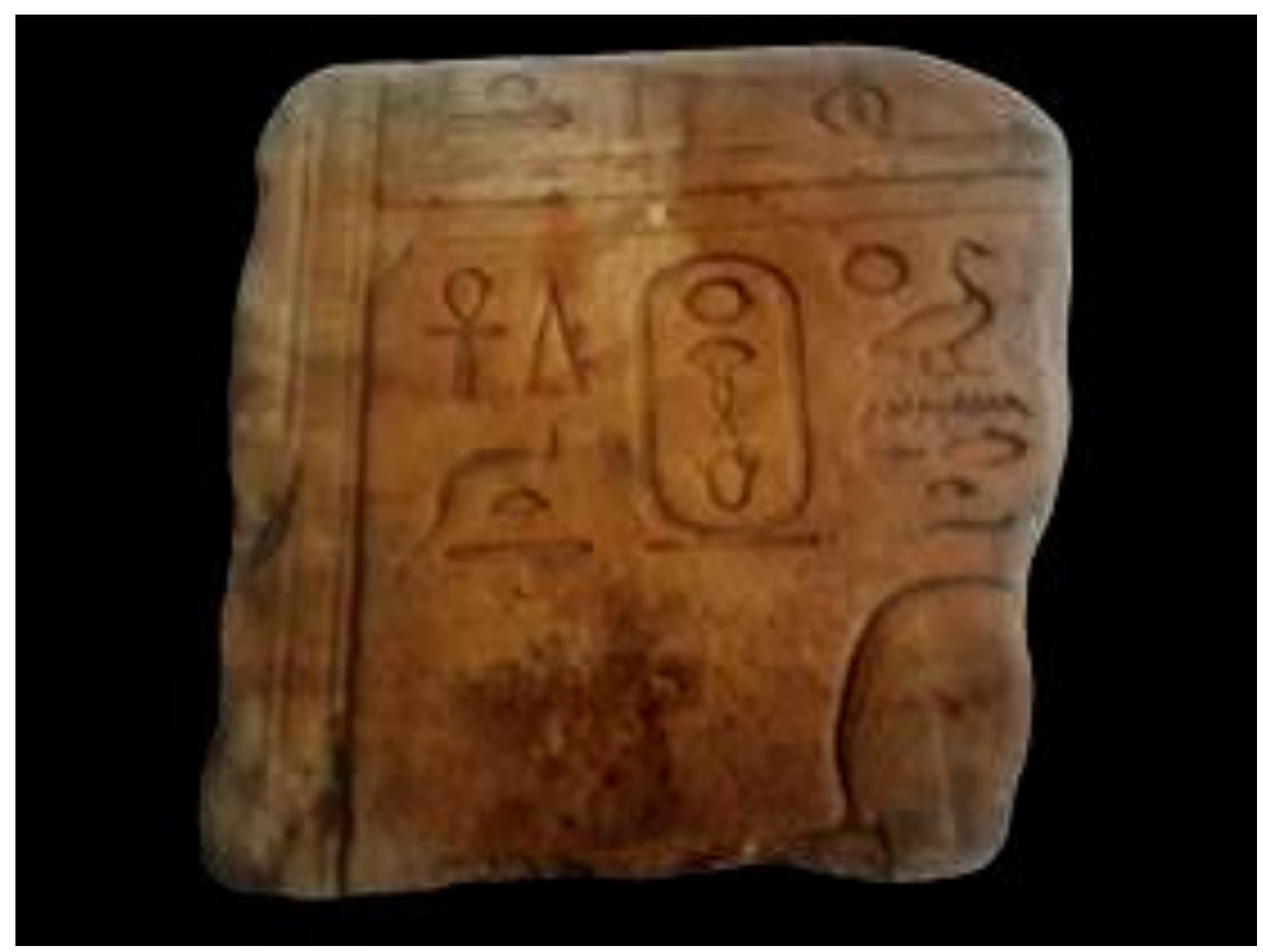

Abb. 8. Die Steinblock von der Vorderseite

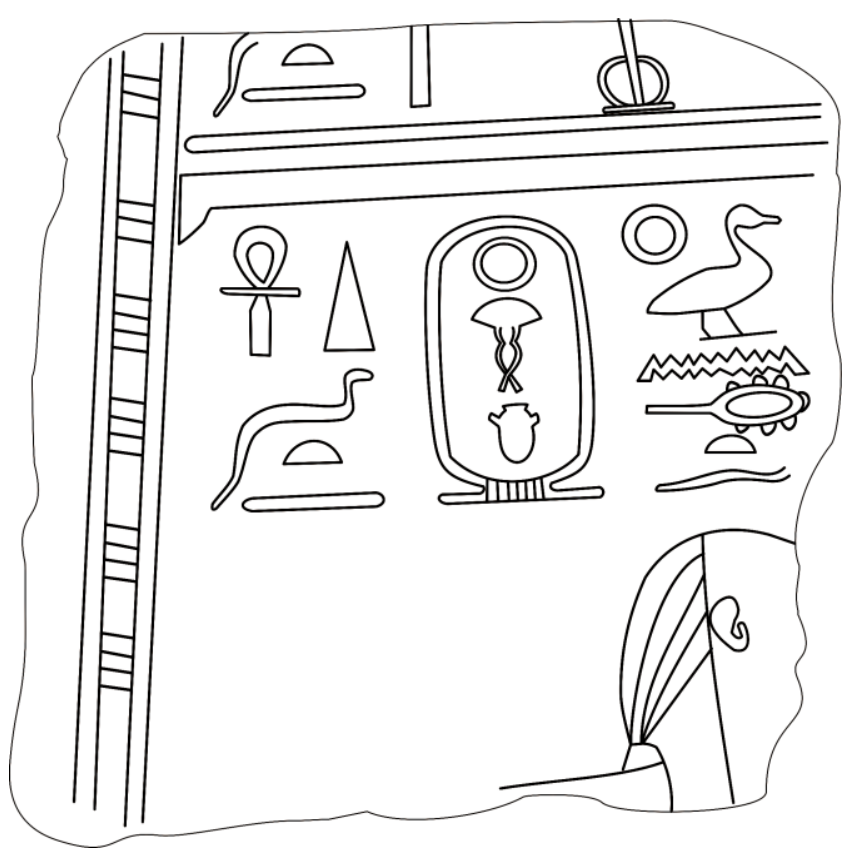

Abb.9 Ein Malen der Steinblock von der Vorderseite 


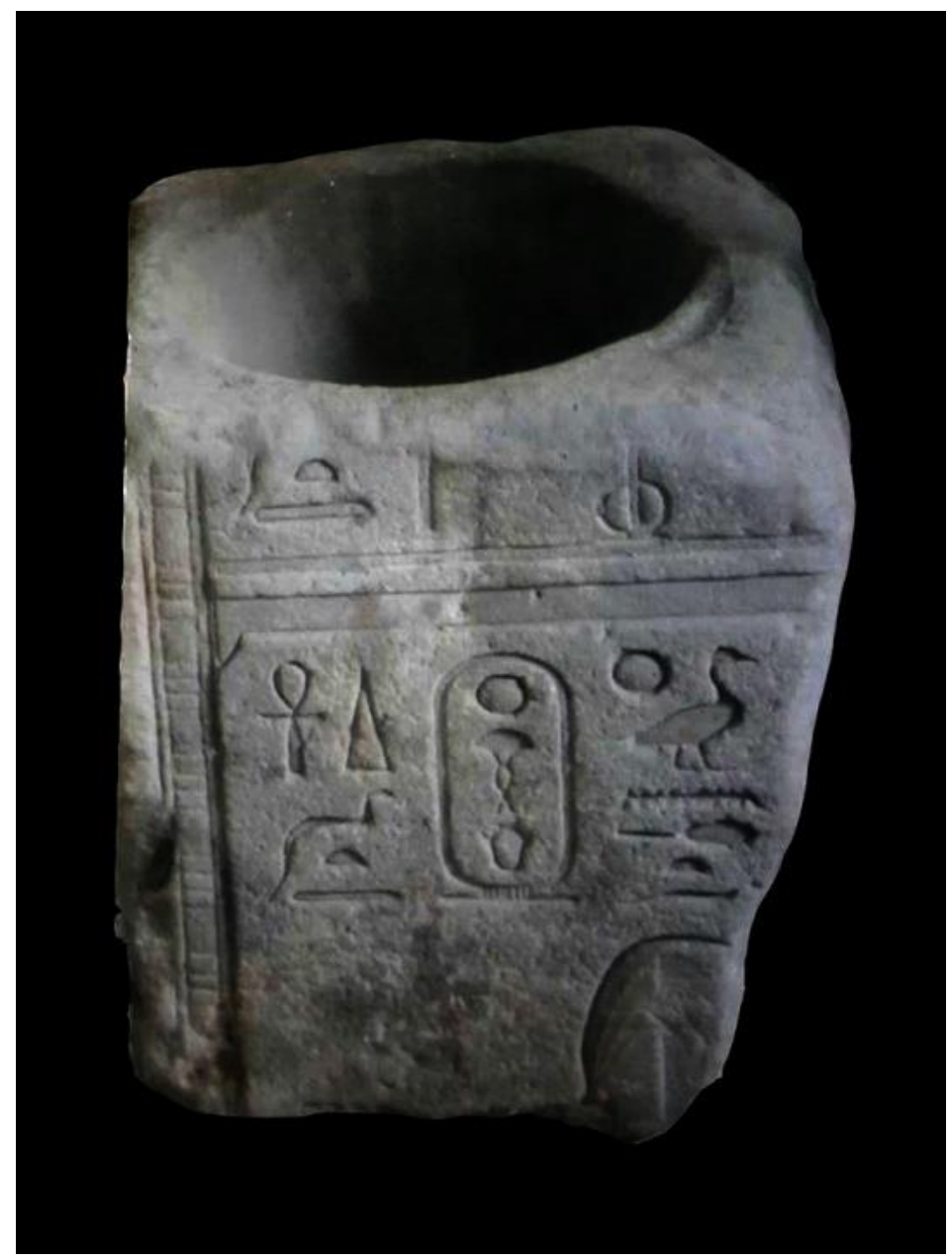

Abb.10

Ein Bild des Steinblocks von oben zeigt den Schlitz im oberen Teil
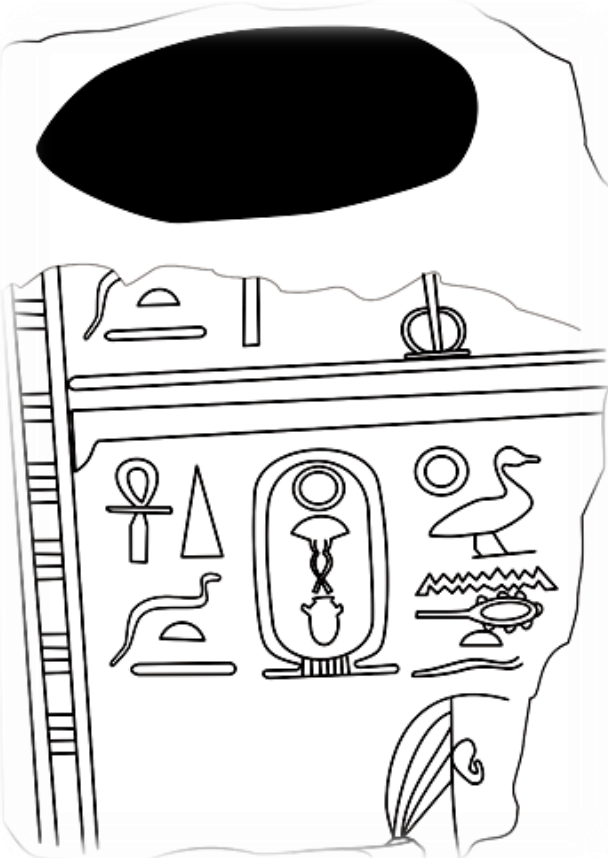

Abb.11 Ein Malen des Steinblocks von Oben zeigt den Schlitz im oberen Teil 
1- Für die Erlaubnis, dieser Architrav publizieren zu dürfen, danke ich den Arbeitern des Ständigen Ausschusses für Antiquitäten

2- M.Moursi, Die Hohepriester des Sonnengottes, $M \ddot{A} S$

26(München,1972).78 Taf. XIII

3- Die Szene der gabenbringenden Götter wird eine Standard-Dekoration der ägyptischen Tempeln nur in der achtzehnten Dynastie. John D. Cooney, Gods Bearing Gifts for the King, The Bulletin of the Cleveland Museum of Art ,Vol. 54, No. 9 (Nov., 1967), 279.

4- Ch.Leitz, Lexikon der ägyptischen Götter und Götterbezeichnungen (2002), IV, 148 .

5- Die erste Zeile ist jetzt verloren. Ich benutze hier das alte Bild des Architravs um die Inschrift zu ergänzen. siehe unten Abb.1=M.Moursi, Die Hohepriester des Sonnengottes, Taf. XIII.

6- Das Wort Snwt wird falsch mit O statt l

7- Wb II;116(18).

8- Ch.Leitz, Lexikon der ägyptischen Götter $\mathrm{V}, 44$.

9-Für Blumen als Opfer siehe: LÄ I, 836; J.Dittmar, Blumen und Blumensträuße als Opfergabe im alten Ägypten, MÄS 43,1986.

10-Bezeichnung des Hapi ;Ch.Leitz, Lexikon der ägyptischen Götter I,580.

11-R.Hannig, Großes Handwörterbuch Ägyptisch-Deutsch (2800950v.Chr.)Kulturgeschichte der Antiken Welt 64 (2006), 502.
12-Ch.Leitz, Lexikon der ägyptischen Götter IV,248.

13- Bezeichnng der Nnwt, Ch.Leitz, Lexikon der ägyptischen Götter IV,95.

14- $\square \square$ liest sich awj siehe : P.Wilson, $A$ Ptolemaic Lexikon: a lexicographical study of the texts in the temple of Edfu, OLA 78, 1997, 131; Wb I, 156.

15-Gans benützt als Opfer um die Vernichtung der Feinde des Gottes symbolisieren. $L \ddot{A}$ II,374.

16- M.Moursi, Die Hohepriester des Sonnengottes, 78, Taf.XIII.

17-John D. Cooney, Gods Bearing Gifts for the King, The Bulletin of the Cleveland Museum of Art ,Vol. 54, No. 9 (Nov., 1967), 279.

18-Für die Erlaubnis, diesen Block publizieren zu dürfen, danke ich Herr Mamdouh Abas, dem Direktor des archäologischen Bereichs, sehr herzlich.

19- Dieses Zeichen besteht aus zwei Teilen, der untere ist das Sn Zeichen und der obere stellt vielleicht ein Fächersymbol oder einen Blütenwedel dar. siehe dazu: H.Kees, Der Opfertanz des ägyptischen Königs (Leipzig,1912) 119 ff. Dieses Zeichen erscheint am meisten in Reliefs mit anderen Zeichen hinter dem König, um ihn zu schützen. Siehe: P.Wilson, $A$ Ptolemaic Lexikon, 1015.

20- Wb III, 410

21-R.Hannig, Großes Handwörterbuch Ägyptisch-Deutsch, 175.

22- Vgl die Steinblöcke von Sais. P.Wilson, The Survey of Sais(Sa el-Hagar) 19972002, $203 \mathrm{ff}$.

23-L.Habachi, Sais and its monuments, ASAE 42, 372. 
